\title{
La práctica docente basada en la investigación de aula con aplicación del componente virtual como herramientas de mejora del aprendizaje en el Programa Educación General Básica I y II Ciclos
}

\author{
Jorge Cartín Obando* \\ María de los Angeles Chavarría Román** \\ Alexis Mata Chacón*** \\ *Asesor Nacional de Estudios Sociales del MEP. Apoyo Académico del Programa \\ Educación General Básica I y /I Ciclos; cartinobandoj@gmail.com \\ **Encargada de Práctica Docente. Programa Educación General Básica I y ll Ciclos; machavarria@uned.ac.cr \\ *** Docente de Matemática del MEP. Profesor Supervisor de Práctica Docente de la UNED; alexismat@gmail.com
}

Recibido: 10 de agosto de 2012

\section{RESUMEN}

Este artículo presenta la innovación educativa, del mejoramiento de las condiciones académicas y técnicas en que se llevan a cabo los procesos de formación de docentes en la UNED, para atender la educación primaria en Costa Rica. El contexto actual demanda un cambio de actitud del docente, basado en el deber de hacer investigación para atender los grandes temas, que están marcando el inicio del siglo XXI. Dichos temas en el ámbito de la educación se expresan en la construcción de conocimientos, la reflexión y la toma de decisiones, orientadas a la mejora cualitativa en el ejercicio profesional de la docencia. Es importante explicitar las bases teóricas que fundamentan el diseño curricular de la práctica docente, basada en la investigación de aula, para incorporar el componente virtual como herramienta de mejora en la formación del alumnado en I y II Ciclos y a partir de esta reflexión, enriquecer nuestras políticas académicas.

\section{PALABRAS CLAVE}

Investigación de aula, práctica docente, TIC, docente como investigador, plan de estudios.
Aceptado: 2 de noviembre de 2012

\section{SUMMARY}

This article seeks to implement educational innovation in regards to the academic and technical circumstances in which formal teacher education processes take place at UNED, in order to address primary education in Costa Rica. Nowadays, the educational environment demands a change of attitude in the teacher, as this person has the duty of researching the significant issues that have marked the beginning of the XXI century. These issues are manifested as knowledge building, reflection and decision making, all of which are oriented to a qualitative enhancement in the professional execution of teaching. It is important to make explicit the theoretical bases that lay foundation to teaching practice curricular design. This curricular design must be based in classroom research and must feature a technological component as a tool for enhancement in alumni education in I and II Cycles. From this insight, we must improve our academic policies.

\section{KEY WORDS}

Classroom research, teaching practice, ICT, teacher as researcher, curriculum. 


\section{INTRODUCCIÓN}

La investigación educativa debe entenderse como un proceso de construcción de conocimiento, el cual se convierte en un instrumento elemental en el proceso de aprendizaje. Asimismo, el estudiante participa en la reflexión de su propia acción en dicho proceso, lo que enriquece la investigación en el aula del docente.

Según Cabrera la investigación en el aula permite el perfeccionamiento del docente teniendo como eje su propia aula (Cabrera, 1996). Este autor aporta una idea clave para explicar la necesidad de tomar en cuenta las propias experiencias del ejercicio docente. El educador debe desarrollar capacidad para vigilar sus propias prácticas de aula, pues de esa forma podrá experimentar el conflicto entre su responsabilidad como educador, respecto de la forma de aprender sus alumnos y su responsabilidad ante la sociedad sobre lo que realmente aprenden. (Elliot, 2005).

La investigación ha tenido una acelerada evolución en las diferentes ciencias y disciplinas, a partir del advenimiento de las tecnologías de la información y la comunicación (TIC). Actualmente, el contexto educativo participa de la evolución tecnológica en los procesos de enseñanza, lo que ha generado un cambio de actitud del docente. Este debe adoptar una actitud investigativa e innovadora dentro del contexto de la sociedad de la información.

El contexto educativo, según lo anterior, debe combinar la práctica docente y el uso de las TIC, de ahí que se hayan implementado las plataformas virtuales como nuevos espacios donde se desarrolla el proceso de enseñanza y aprendizaje, especialmente, en la modalidad de educación a distancia.

Este artículo promueve la discusión acerca de las bases de la investigación educativa, desde sus premisas teóricas y la reflexión sobre la necesidad de innovar el proceso de enseñanza y aprendizaje, mediante la utilización de los espacios virtuales. Se pretende articular ambos elementos en el contexto del cambio conceptual y metodológico de la práctica docente.

\section{METODOLOGÍA}

Para el desarrollo de este artículo se hizo una amplia consulta bibliográfica con el propósito de promover la presentación de un futuro curso de práctica docente, el cuál servirá como un insumo al mismo en el nivel de bachillerato del Programa de I y II Ciclos. La recopilación, análisis y síntesis de los documentos, permitió la fundamentación de los siguientes apartados: I- La investigación de aula y el rol del docente como aprendiz, II- La investigación de aula como proceso evaluativo para la mejora continua de la práctica docente, III- El uso de la plataforma virtual como apoyo a la investigación en el aula y IV- La investigación de aula y el componente virtual como elementos para el cambio epistemológico en la práctica docente del Programa Educación General Básica I y II Ciclos.

\section{DESARROLLO}

\section{- La investigación de aula y el rol del docente como aprendiz: antecedentes}

La necesidad de remozar la Práctica Docente a partir del fortalecimiento de la investigación, responde a los cambios actuales en el campo social, económico, político, ético y pedagógico en el marco de un mundo globalizado. El docente se enfrenta a retos y demandas que debe asumir en busca de la mejora del proceso de formación docente en la educación primaria.

\section{¿Qué es la investigación de aula?}

La investigación de aula se concibe como la mirada rigurosa y científica que pretende construir, explicar o comprender el fenómeno educativo. El estudio constante del hecho 
educativo en el aula resulta un proceso de aprendizaje para el docente, quien debe asumir una actitud objetiva y reflexiva de su propia labor. La problematización sistemática y crítica del quehacer docente, en búsqueda de explicaciones o interpretaciones del fenómeno educativo, permite la cualificación de la profesión y, por tanto, el perfeccionamiento de su ejercicio. (Salcedo, 2001).

La investigación de aula, en congruencia con lo anterior, promueve la formación de docentes que se preguntan acerca de lo que sucede en el entorno, sujetos activos, polivalentes y con una actitud de indagación científica, basada en el tratamiento de problemas mediante la pedagogía de la pregunta, el razonamiento divergente, que se perfecciona en la práctica y que tiene unos fines educativos determinados (Porlán, 1999).

Existe diversidad de enfoques que se refieren a la comprensión del fenómeno educativo. Dichos enfoques permiten situar la relación entre docente y estudiante desde una perspectiva cualitativa y crítica, en función del cambio del rol de la figura docente. Esta figura, tradicionalmente, se había anclado en una concepción transmisiva de los conocimientos, así como una actitud pasiva y receptora del alumnado durante la adquisición de esos saberes.

Se pueden mencionar algunos enfoques tales como el etnográfico, la investigación-acción, la fenomenología y la condicionalidad situada, los cuales posibilitan una lectura comprensiva, más acorde con las necesidades e intereses del alumnado y la construcción continua de los aprendizajes.

No debe perderse de vista que los enfoques de comprensión del hecho educativo, buscan una resignificación de la subjetividad de los conocimientos, habilidades y destrezas, que poseen tanto los estudiantes como los maestros. El rol tradicional de la figura del docente como depositario absoluto de los conocimientos, debe modificarse por una figura como aprendiz en el aula. Este último aspecto pone de relieve otro detalle importante, el cual consiste en convertir al aula como centro del debate de ideas y por qué no, de una pedagogía democrática y deliberativa por oposición a una pedagogía rígida y autoritaria.

Se presenta, a continuación, un cuadro comparativo acerca del rol del docente como aprendiz y la figura del docente tradicionalista.

Cuadro 1. Cuadro comparativo: rol del docente aprendiz y docente tradicionalista

Docente como aprendiz

Ejerce un rol activo como orientador del proceso de enseñanza y aprendizaje.

Planifica estrategias centradas en la negociación de significados entre los estudiantes.

Concibe al estudiante como sujeto activo en la construcción de conocimientos.

Aprende conjuntamente con el estudiantado poniendo en práctica la investigación en el aula, mediante la construcción conjunta del conocimiento.

La subjetivad de los alumnos y docentes en la construcción del conocimiento adopta un rol decisivo.
Docente tradicionalista

Mantiene un rol instructivo en la organización del hecho educativo.

Las estrategias de enseñanza se basan en la transmisión de conocimientos.

Concibe al estudiante como un receptor pasivo de la información.

El saber y los conocimientos no se investigan, no se construyen, porque ya vienen determinados en los libros de texto y en diversas fuentes del saber.

El conocimiento ya está estructurado y proviene de los saberes establecidos de la disciplina, de ahí su carácter objetivo.

Fuente: Elaboración propia de los autores 
Se deduce, del cuadro anterior, diferencias significativas entre ambos tipos de rol ejercido por el docente. Lo importante es tener presente que la investigación de aula constituye un salto cualitativo y toma en cuenta el rol activo de aprendizaje continuo de la figura docente. De esta forma, el maestro aprende de las necesidades, inquietudes e intereses de sus estudiantes, los cuales se convierten en verdaderos soportes que validan el alcance de las prácticas pedagógicas puestas en marcha.

De acuerdo con Grundy (1998), Zabalza (2000) y Stenhouse (2007), los momentos de investigación de aula constituyen un ciclo que se puede sintetizar de la siguiente forma.

Debe resaltarse que el contexto complejo en el que se desenvuelve el hecho educativo en la sociedad actual, la polivalencia de los saberes que deben poseer docentes y estudiantes, fomentan la necesidad de incorporar continuamente la investigación de aula, como una forma de actualización y profesionalización continua del docente.
- La Investigación de aula como proceso evaluativo y de mejora continua de la práctica docente

Es preciso que el docente tenga claro el concepto de la acción educativa mediada, para cumplir con las perspectivas de la investigación educativa y sus fundamentos. Esta labor debe estar en estrecha relación con los procesos evaluativos, con miras al mejoramiento de la calidad de la formación docente.

La acción educativa mediada asociada a procesos evaluativos, busca promover las habilidades y destrezas de la figura docente, centradas en direccionar el proceso de enseñanza-aprendizaje. Relacionado a lo anterior, en el quehacer del docente se vivencian interrogantes, asociadas a un compromiso social, profesional y personal del educador con los alumnos y la sociedad en la que se vive.

Una de las maneras de solucionar estas preguntas, es la reflexión teórica-práctica, para

Cuadro 2. Ciclo de la investigación de aula

\begin{tabular}{|l|l|}
\hline \multicolumn{1}{|c|}{ Momentos de la investigación de aula } & \multicolumn{1}{c|}{ Descripción } \\
\hline Observación & $\begin{array}{l}\text { La realiza el docente enfatizando en los estilos de } \\
\text { aprendizaje de sus estudiantes. }\end{array}$ \\
\hline Diagnóstico & $\begin{array}{l}\text { Es una valoración inicial de los estilos, ritmos de apren- } \\
\text { dizaje y el estado general de las habilidades y destre- } \\
\text { zas del estudiantado. }\end{array}$ \\
\hline Sistematización de información & $\begin{array}{l}\text { Permite un marco general de criterios acerca de las } \\
\text { fortalezas y debilidades que los (as) docentes van do- } \\
\text { cumentando sobre la práctica pedagógica }\end{array}$ \\
\hline Propuesta emergente de cambio & $\begin{array}{l}\text { Herramientas teórico-metodológicas para modificar } \\
\text { las condiciones del proceso de enseñanza-aprendizaje }\end{array}$ \\
\hline Revisión de la teoría y de la metodología aplicada & $\begin{array}{l}\text { Relación que se desarrolla entre la teoría y la práctica a } \\
\text { partir de las soluciones implementadas }\end{array}$ \\
\hline Nueva teoría surgida de la práctica & $\begin{array}{l}\text { Permite el reinicio del ciclo de la investigación de aula a } \\
\text { partir de la realimentación entre teoría y práctica y pro- } \\
\text { mueve la innovación en la enseñanza y el aprendizaje. }\end{array}$ \\
\hline
\end{tabular}

Fuente: Elaboración propia de los autores a partir de los aportes de Grundy (1998), Zabalza (2000) y Stenhouse (2007). 
plantear alternativas de solución al accionar de la práctica docente. Retomando líneas anteriores, el docente es una figura práctico-reflexiva, que debe observar e indagar las dinámicas de trabajo de su grupo, las interacciones entre sus alumnos y también las relaciones cognitivas y socio afectivas docente-educando.

La acción práctico-reflexiva en la docencia viene acompañada de un importante componente normativo, aunado a ello también deben reunirse conocimientos de tipo instrumental. Existen técnicas de trabajo para la recolección de información que se consideran necesarias y pertinentes para alcanzar el propósito de construir teorías a partir de la práctica. Es importante hacer un breve recuento de este tipo de técnicas.

La aplicación de estas técnicas de estudio puede que permitan al estudiante el mejoramiento de su rendimiento académico y fortalecer sus estrategias de aprendizaje que le lleven al éxito y a un amplio nivel de motivación. Las técnicas seleccionadas y llevadas a la acción por el grupo y por la figura docente, deben ayudar a explorar o en nuestro caso en estudio, a evaluar las múltiples facetas del propio estudiante, su autoestima, sus hábitos y otros.

Para el docente es importante tener en consideración técnicas como el cuestionario, entrevista, grupo focal, observación directa, entre otras, que inciden favorablemente en el desempeño académico del alumnado. Tanto en el ámbito de la práctica docente, como en el ejercicio profesional de la docencia, siempre guarda un lugar importante la aplicación de un diagnóstico inicial al grupo de estudiantes que se tiene a cargo. Las distintas secciones del cuestionario y entrevistas, permiten la obtención de información tanto cuantitativa como cualitativa, que hacen posible la contextualización de las necesidades e intereses del alumnado, de forma tal que esto sirva como insumo a la planificación educativa en el salón de clases.

No debe de olvidarse que el análisis de los resultados, bajo la aplicación de la metodología estadística, permite el nivel descriptivo como de correlación de variables, necesarios para hacer una planificación educativa más pertinente y realista, que posibilita la evaluación diagnóstica de entrada.

Es con estos resultados y desde un enfoque de la evaluación alternativa, que se intenta comprender qué asuntos son de verdad importantes $y$, por ende, necesarios de transformar, teniendo un significado formativo. Esto permite la mejora de los procesos didácticos en el aula en determinado tema dado su carácter participativo, debido a que los involucrados hacen parte de la investigación. Es decir, estudiantes y docentes participan en el equipo, que con sus intervenciones realimentan la evaluación.

Por otra parte, la investigación educativa puede contar con otra técnica interesante: "grupo focal", que de acuerdo con Hernández, Fernández y Batista (2006), es una de las formas de los estudios cualitativos en el que se reúne a un grupo de personas para indagar acerca de actitudes y reacciones frente a una situación dada, donde la técnica o procedimiento, indica que los investigadores deben valerse de experiencias subjetivas. Dicha técnica viene a llenar un vacío dejado por los cuestionarios que presentan limitaciones por la constante frecuencia de preguntas cerradas que obligan y sesgan las respuestas de los participantes.

En cuanto a la técnica de la "observación directa" manejada con propiedad y con los registros basados en el uso de matrices que cotejan lo visualizado, se logra un acercamiento de aquellos códigos extra lingüísticos que permiten también conseguir datos relevantes para mejorar el proceso de enseñanza-aprendizaje.

Una vez hecho este breve recuento de la posible utilización de técnicas para la obtención de datos e información para la toma de decisiones, con miras al mejoramiento del accionar docente, resulta importante decir que la evaluación no se limita a un diagnóstico de entrada, sino, que se realiza de una forma procesal. Es decir, la evaluación debe conceptuarse como una 
herramienta de mejora continua del aprendizaje estudiantil y del propio docente.

Para finalizar este apartado debe plantearse que al tomar conciencia de las posibilidades y limitaciones que puede tener el proceso de enseñanza y aprendizaje implementado, el o la docente como aprendiz de su propio proceso de mediación pedagógica logra interiorizar el cambio, la mejora y la innovación, como resultado de la atención de las necesidades, los intereses, los estilos, los niveles y los ritmos de aprendizaje del estudiantado.

\section{- El uso de la plataforma virtual como apoyo a la investigación en el aula}

Las tecnologías de uso educativo son utilizadas para la enseñanza presencial o a distancia. La educación ha sido una de las más beneficiadas con el advenimiento de las nuevas tecnologías, especialmente las relacionadas con la Web 2.0, en donde se generan y se comparten contenidos.

Los diferentes servicios brindados en internet a los estudiantes se han tratado de estructurar en un único entorno, a través de numerosas empresas.

"Los docentes, las instituciones educativas públicas y privadas que apuestan por la enseñanza flexible, abierta y a distancia agradecen la posibilidad de disponer de una amplia gama de entornos virtuales de aprendizaje diseñados para la gestión y desarrollo de cursos y programas". (García, L. 2006).

Algunas de las plataformas de generación de contenidos en línea que se destacan y que pueden integrarse a los entornos educativos son: blogs, wikis, redes sociales y colaboratorios. Estos últimos simplifican el intercambio y acceso de insumos entre profesores-académicos-estudiantes, como documentos científicos, proyectos, reportes, conferencias, papers, clases, tareas, estudios, bases de datos, entre otros. (Cobo, 2007).

El contexto del conocimiento actual presenta diferentes plataformas en el mercado, con funciones distintas ya que cubren diferentes necesidades, de las cuales algunas se utilizan en educación. Estos insumos educativos constituyen "una especie de espacios virtuales que por medio de recursos tecnológicos contienen materiales con un contenido específico que permite la construcción y transferencia de conocimientos entre los miembros de un grupo" (Burgos, 2007).

Igualmente, Delgado (2005) las considera sistemas que proveen todos o la mayor parte de los servicios que están a disposición de los estudiantes en un campus universitario real. Definición que va enfocada hacia la educación a distancia en que el profesor y el alumno se relacionan por medios tecnológicos.

La selección de una plataforma educativa gira en torno al aprendizaje significativo y colaborativo, y no es una tarea sencilla. Por tal motivo la interactividad, sostiene Delgado (2005), es fundamental porque permite una acción recíproca entre estudiantes y docentes, para que cada participante se transforme en un sujeto capaz de comunicarse con el docente tutor para alcanzar los objetivos del curso.

Además, el autor en mención indica que este medio virtual debe tener una interfaz amigable; instrumentos de evaluación formativa, aprendizaje colaborativo, acceso a cualquier computadora, compatible con diferentes navegadores, equipos actualizados y un manejo ágil de las inscripciones y perfiles de entrada de los usuarios del sistema.

El estudiante de la Carrera de Educación General Básica I y II Ciclos, si bien es estudiante de un modelo de educación a distancia, sigue siendo orientado por una actividad docente presencial.

No obstante, el futuro docente trabajará con estudiantes que cambian aceleradamente con el transcurrir del tiempo en un mundo cada vez más globalizado, en el cual las tecnologías de la información y comunicación están configurando los espacios educativos. En este contexto, el estudiante que ha tenido una educación a 
distancia o algún tipo de e-learning (aprendizaje a distancia vía electrónica) tendrá alguna ventaja de percibir como algo natural que vive en una sociedad altamente digitalizada.

Los efectos sociales de las TIC ya empiezan a ser visibles en las aulas. Por ejemplo, los estudiantes utilizan internet como herramienta de investigación o como medio de interacción social. El futuro docente debe ser flexible para adaptarse a situaciones de continuo cambio, debe estar preparado y comprender todos los elementos y experiencias de las TIC.

El docente investigador al proyectarse al futuro, debe tener sumo cuidado porque

"no solo puede llevarlo a interpretar los datos en determinado sentido, sino también, y de manera totalmente inconsciente, a inducir a los participantes a comportarse como él espera que lo hagan" (Giroux y Tremblay, 2009).

El docente investiga para conocer y para enseñar, asimismo, también puede estudiar problemas específicos de su salón de clases para encontrar un adecuado desempeño y mejorar su acción didáctica.

De acuerdo al trabajo docente en el aula, existen dos tipos de investigación: la básica y la aplicada. La primera mejora el conocimiento del fenómeno estudiado y la segunda orienta la acción pedagógica.

De conformidad con las diferentes corrientes del pensamiento, estas se han polarizado en dos enfoques principales: el cuantitativo y el cualitativo. Si ambos enfoques convergen en un mismo estudio se les estaría denominando enfoque integrado multimodal o enfoques mixtos (Hernández, Fernández y Batista, 2006).

El docente en el aula puede utilizar ambos enfoques. Si utiliza el cualitativo para estudiar un fenómeno lo haría con énfasis en la comprensión y si utiliza el enfoque cuantitativo lo haría con énfasis en la medición y el análisis de datos con cifras (Giroux y Tremblay, 2009).
Todo docente debe formarse en investigación, como parte de su currículo escolar. La investigación escolar emerge desde el aula y debe dar rienda suelta a su curiosidad, al sentido de indagación que todos traemos desde niños, para enriquecer el conocimiento y así ser parte del engranaje para modificar y actualizar sus prácticas educativas y mejorar su competencia profesional.

\section{- La investigación de aula y el componente virtual como elementos para el cambio epistemológico en la práctica docente del Programa Educación General Básica I y II Ciclos}

La educación hoy en día sigue valorándose como una poderosa herramienta de cambio de la sociedad. Tiene como intención promover habilidades investigativas para sentar las bases de la transformación de la práctica docente.

En el ámbito de bachillerato en I y II Ciclos, se propone en la nueva estructura del plan de estudios del Programa, la Práctica Docente II como opción de graduación relacionada con la investigación de aula y el componente virtual, en que se busca una reorientación epistemológica del quehacer de quiénes aspiran a convertirse en docentes. Esta visión implica un cambio en el rol de la figura docente que pasa de ser un trasmisor de información a convertirse en aprendiz junto a sus estudiantes. Significa un redimensionamiento de la forma en que se construyen los saberes dentro y fuera del aula, con el fin de superar el mero consumo de conocimientos para pasar a nuevas formas de interpretación de la realidad.

Este replanteamiento de la función docente va paralelo a las transformaciones tecnológicas que se vienen desarrollando en el marco de la denominada sociedad de la información y el conocimiento. La ampliación en la cobertura informática y de redes telemáticas, indiscutiblemente viene dando un viraje que también influye en la modificación de las 
relaciones entre el ejercicio de la docencia y la formación de los educandos.

Es prioritario pensar que la forma en que se impartirá la práctica docente estará influida por los componentes de la investigación de aula y el uso de las plataformas virtuales. Esta situación, ampliará el espectro o el radio de acción de la oferta educativa que permitirá una mayor presencia en los diferentes rincones del país.

En el ámbito del rediseño curricular del plan de estudios del Programa de Educación General Básica I y II Ciclos, resalta la investigación como un recurso didáctico en la formación de docentes. Este enfoque está íntimamente relacionado con la formación que concibe que el aprendizaje se adquiere mediante un proceso de experimentación y reflexión colectiva.

El propósito de la práctica docente II basada en la investigación de aula y la aplicación del componente virtual, busca la formación de una figura docente que se apoyará en la innovación educativa, como respuesta a los problemas en el aprendizaje que han sido detectados en la práctica pedagógica llevada a cabo por docentes y estudiantes.

La investigación educativa puede asumir tres roles en la formación docente; por un lado, la investigación en forma de metodología, como una materia en el currículo para formar a docentes-investigadores. Por otro, a través del desarrollo de investigaciones por parte de los formadores de formadores (los profesores de los centros de formación docente) para mejorar su docencia; y por último, la investigación como recurso didáctico en la formación del docente.

\section{CONCLUSIONES}

El rediseño del plan de estudios para la Carrera de Educación General Básica de ly ll Ciclos se encuentra justificado en el proceso de autoevaluación desde sectores académicos, empleadores y el perfil del estudiante de educación primaria.
Por otra parte, la investigación educativa debe seguir tres roles en la formación docente. En primera instancia, la investigación educativa, la cual comienza desde las aulas. Seguidamente, la práctica docente acorde con las necesidades del contexto educativo costarricense.

Finalmente, el rol del educador debe gestarse a partir del currículo de formación de formadores, con el fin de fomentar el desarrollo de competencias teórico- prácticas, basadas en la investigación de aula como parte de la formación continua para la mejora de la profesión. Este es el fin de la preparación de todo docente, con el propósito de que pueda llevar no solo conocimiento, sino entusiasmo y habilidades propias de los nuevos ciudadanos inmersos en el mundo de la sociedad y la información, para lo cual las TIC son recursos idóneos.

El desarrollo de los cursos virtuales, si bien es cierto, no es la solución total a los problemas educativos, es un medio para lograr que los y las estudiantes reciban una educación más democrática e inclusiva, por ello es necesario avanzar planificada y progresivamente hacia la virtualización. La difusión y la implantación del uso de las nuevas tecnologías en el entorno educativo, es un apoyo para la consecución de los fines sociales de la educación tal y como lo enuncia la Ley Fundamental de Educación vigente desde 1957.

\section{REFERENCIAS}

Burgos A., J. y Lozano R., A. (2007). Tecnología Educativa en un modelo de educación a distancia centrado en la persona. México: Limusa.

Cabrera, G.; Rodríguez, R.; Fernández, J. (1996). La estrategia de triangulación en la investigación acción: materiales didácticos en el aula de Física. Actas del $X$ Congreso de la Didáctica de la Física, 362-39o.

Cobo Romani, C. y Pardo Kuklinski, H. (2007). Planeta Web 2.o. Inteligencia colectiva o medios fastfood. México: Flacso Recuperado de http:// www.flacso.edu.mx 
Delgado, K. (2005). Las plataformas en la educación a distancia. Revista Iberoamericana de Educación. Recuperado de http://www.rieoei.org/ deloslectores/1300Delgado.pdf

Elliott, J. (2005). El cambio educativo desde la investigación-acción. Madrid: Ediciones Morata.

García A., L. (2001). La educación a distancia. De la teoría a la práctica. Madrid: Ariel.

Giroux, S. y Tremblay, G. (2009). Metodología de las ciencias humanas. México: IEPSA

Grundy, S. (1998) Producto o praxis del currículum. Madrid: Ediciones Morata.

Henández, R., Fernández, C y Baptista, P. (2006). Metodología de la Investigación. (4ta. ed.). México: McGraw-Hill/Interamericana Editores.
Porlán, R. (1999). El currículum en acción.( Mimeografiado) Universidad de Barcelona.

Salcedo, R (2001). La investigación en el aula y la innovación pedagógica .IDEP: Bogotá.

Stenhouse, L. (2007). La investigación como base de la enseñanza. Selección de textos por Rudduck y Hopkins. Madrid: Ediciones Morata.

UNED, (1984). Carrera Ciencias de la Educación con Énfasis en I y II Ciclos (Macroprogamación), San José, Costa Rica, (mimeografiado).

Zabalza, Miguel. (2000). Diseño y Desarrollo Curricular. Madrid, Ediciones Narcea. S. A. 
